\title{
Senken Statine das Erkrankungsrisiko?
}

Fragestellung: Beeinflusst die Einnahme von Statinen das Risiko, an einem Gliom zu erkranken?

Hintergrund: Die Ursachen der Entstehung von Gliomen sind bis auf eine genetische Komponente und den Einfluss von Strahlenexposition im Kindesalter unbekannt. Experimentelle Studien und eine Fall-Kontroll-Studie legen nahe, dass der Einsatz von Statinen das Risiko für eine Gliomerkrankung reduzieren könnte.

Patienten und Methodik: Die Autoren führten eine nationale Fallkontrollstudie in Dänemark durch, in die sie alle Patienten zwischen 20 und 85 Jahren mit der Diagnose eines histologisch gesicherten Glioms zwischen 2000 und 2009 einschlossen. Als Kontrollgruppe zogen sie sieben bis acht alters- und geschlechtsangepasste Kontrollen heran. Eine Statineinnahme seit 1995 wurde als kurzzeitig (unter fünf Jahren) oder langzeitig (mehr als fünf Jahre) sowie nach der Intensität der Statineinnahme klassifiziert. Es wurden nur Patienten ohne dem Gliom vorausgehende Tumorerkrankung eingeschlossen.

Ergebnisse: 2.656 Patienten mit Gliomen wurden mit 18.480 Kontrollpersonen verglichen. Bei Langzeit-Statineinnahme war das Risiko einer Gliomerkrankung gegenüber Personen, die nie Statine einnahmen, reduziert. Das Risiko war auch von der Intensität der Einnahme abhängig. Die Assoziation war bei Männern unter 60 Jahren deutlicher als bei Männern über 60 Jahren. Die Beziehung war bei Frauen unter 60, nicht aber bei Frau-

Gaist D, Andersen L, Hallas J, et al. Use of statins and risk of glioma: a nationwide case-control study in Denmark. Br J Cancer im Druck en über 60 Jahren nachweisbar.

Schlussfolgerung: Eine Statineinnahme könnte das $\mathrm{Ri}$ siko von Gliomerkrankungen reduzieren.

\section{Weitere Infos auf \\ springermedizin.de}

Palliativversorgung in der Neuroonkologie (3668084).

Die palliative Versorgung von Menschen mit fortgeschrittenen Hirntumoren ist herausfordernd, da oft ausgeprägte kognitive, emotionale und körperliche Veränderungen bestehen. Symptomerfassung wie auch Kommunikation gestalten sich daher oft schwierig und andersartig. Diesen Artikel finden Sie, indem Sie die in Klammern gesetzte ID-Nummer in das Suchfeld auf dem Springer Medizin-Portal eingeben.

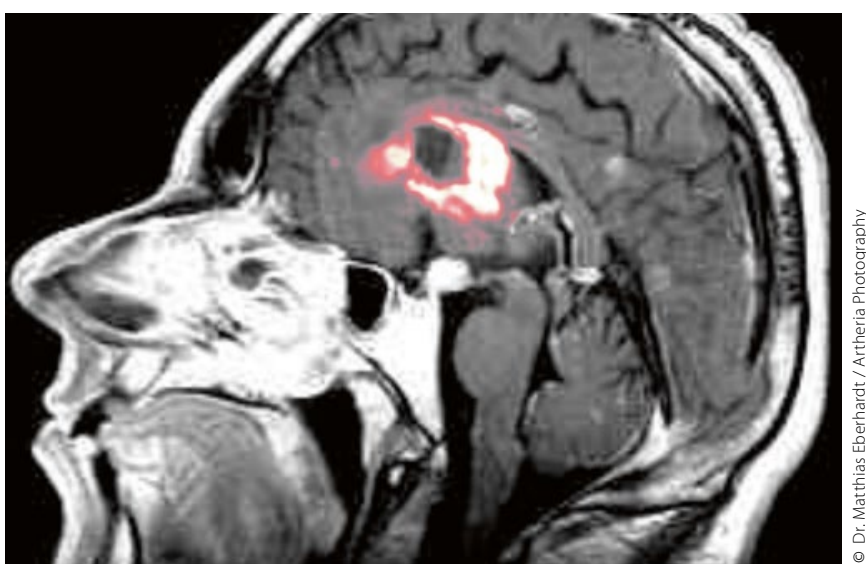

1 Die Tumorinzidenz unter Statinen wird intensiv diskutiert.

\section{- Kommentar von Professor Michael Weller}

\section{Spannende Ursachensuche}

Es handelt sich um eine methodisch saubere Studie, die nahelegt, dass eine längerfristige und intensive Einnahme von Statinen das Erkrankungsrisiko bezüglich Gliomen reduziert. Die Autoren diskutieren angemessen mögliche interferierende Faktoren, die dieses Ergebnis erklären könnten. Dass Statine nach Schlaganfall eingesetzt werden und bei solchen Patienten ein Gliom aufgrund der Bildgebungen häufiger diagnostiziert wird, würde gegen den beobachteten Effekt sprechen. Andererseits ist die Statineinnahme auch mit Diabetes assoziiert, und Diabetes wiederum mit Krebserkrankungen. Auch dies würde eine gegenläufige Verfälschung bewirken.

In Untersuchungen an Zellkulturen zeigten Statine bei hohen Konzentrationen eine zytotoxische Wirkung auf Gliomzellen. Es scheint aber zu spekulativ, bei diesen Beobachtungen aus der Zellkultur die Ursache für die hier beobachtete, interessante Assoziation zu suchen. Weitere Untersuchungen zur Aufklärung dieses Zusammenhangs dürfen mit Spannung erwartet werden.

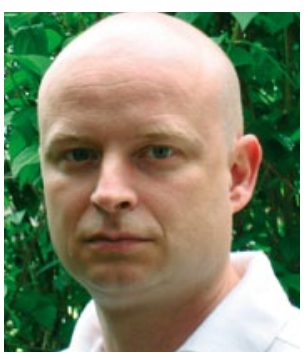

Prof. Dr. med. Michael Weller, Zürich, Schweiz

Direktor der Klinik für Neurologie, Universitätsspital Zürich E-Mail:michael.weller@usz.ch 